\title{
Thermodynamical property of entanglement entropy and deconfinement phase transition
}

\author{
Mitsutoshi Fujita $\odot,{ }^{1, *}$ Song He $\odot,{ }^{2,3, \dagger}$ and Yuan Sun ${ }^{1, *}$ \\ ${ }^{1}$ School of Physics and Astronomy, Sun Yat-Sen University, Guangzhou 519082, China \\ ${ }^{2}$ Center for Theoretical Physics, College of Physics, Jilin University, \\ Changchun 130012, People's Republic of China \\ ${ }^{3}$ Max Planck Institute for Gravitational Physics (Albert Einstein Institute), \\ Am Mühlenberg 1, 14476 Golm, Germany
}

(Received 12 July 2020; accepted 15 November 2020; published 15 December 2020)

\begin{abstract}
We analyze the holographic entanglement entropy in a soliton background with a background gauge field and derive a relation analogous to the first law of thermodynamics. The confinement/deconfinement phase transition occurs due to the competition of two minimal surfaces. The entropic $\mathrm{C}$ function probes the confinement/deconfinement phase transition. It is sensitive to the degrees of freedom smaller than the size of a spatial circle. When the background gauge field becomes large, the entropic $\mathrm{C}$ function becomes nonmonotonic as a function of the size and does not satisfy the usual c-theorem. We analyze the entanglement entropy for a small subregion and the relation analogous to the first law of thermodynamics. For the small amount of a background gauge field, the excited amount of the entanglement entropy decreases from the ground state. It reflects that confinement decreases degrees of freedom. We finally discuss the second order correction of the holographic entanglement entropy.
\end{abstract}

DOI: $10.1103 /$ PhysRevD.102.126019

\section{INTRODUCTION}

The entanglement entropy of a subsystem becomes a nonlocal quantity in contrast to correlation functions in quantum field theories [1,2]. The entanglement entropy is divergent at the critical point for quantum critical phase transitions and becomes an order parameter [3]. It captures geometric discernment of field theories such as an area law [4]: the entanglement entropy defined in a subregion depends on properties of a shared boundary and looks like the black hole entropy.

The Ryu-Takayanagi formula proposes the holographic dual of the entanglement entropy [5-7], which is the area of minimal surfaces. It is a powerful tool to analyze strongly coupled systems. It has been the order parameter of the confinement/deconfinement phase transition in a confining gauge theory $[8-13] .{ }^{1}$ The phase transition occurs due to the competition of two minimal surfaces [8]. After the

\footnotetext{
*fujita@mail.sysu.edu.cn

hesong@jlu.edu.cn

‡sunyuan6@mail.sysu.edu.cn

${ }^{1}$ The holographic entanglement entropy (HEE) also probes holographic superconductor phase transitions [14-20].

Published by the American Physical Society under the terms of the Creative Commons Attribution 4.0 International license. Further distribution of this work must maintain attribution to the author(s) and the published article's title, journal citation, and DOI. Funded by SCOAP ${ }^{3}$.
}

phase transition (large length), the entanglement entropy turns out to be trivial in the confined phase at the infrared red limit. That is, there is no correlation with energy smaller than the mass gap. The phase transition is analogous to the finite temperature deconfinement transition: the finite part of the entanglement entropy becomes $O\left(N^{2}\right)$ at small length and $O(1)$ at large length. The phase transition in gravity duals was connected to those of large $N_{c}$ confining field theories [9] and pure $S U(3)$ Yang-Mills theory [21]. Consistent gravity dual of a confining theory is expected to exhibit this phase transition.

The entanglement entropy for excited states has been attracting attention. The entanglement entropy in the small region satisfies a relation similar to the first law of thermodynamics [22],

$$
T_{\text {ent }} \Delta S_{A}=\Delta E_{A},
$$

where $\Delta E_{A}$ is the increased amount of energy in the subregion and $\Delta S_{A}$ is the increased amount in excited states compared with the ground state of a CFT. $T_{\text {ent }}$ is called the entanglement temperature. This relation has been investigated in many holographic models dual to field theories at finite temperature $[23,24]$. There are very extensive investigations [25-34] on the first law like relation of the holographic entanglement entropy in various cases.

The second order correction to the holographic entanglement entropy has been studied by [35]. In the paper, 
authors rewrite the first law like relation of entanglement entropy in terms of the relative entropy [35]. They calculated the first law like relation of entanglement entropy with spherical entanglement surface up to second order and they also took the deformation of entanglement surface into account. For spherical entanglement surface, authors extend the first law relation to higher order and give insights to use boundary information [36] to reconstruct the bulk geometry. However, it is very difficult to study the second order correction to the holographic entanglement entropy for generic entanglement surface, for example, strip case [37] and so on. In Ref. [37], the authors have studied the strip entanglement surface up to the second order corrections from gravitational background without taking the shape deformation of the entanglement surface into account. For higher derivative gravity, the dictionary of the holographic entanglement entropy has to be changed in terms of [38]. In higher derivative gravity theories, the second order of holographic entanglement entropy becomes very complicated. In the literature, the authors [39-42] have studied the similar first law like relation of the entanglement entropy in various situations. Further, the corresponding holographic entanglement chemistry is investigated in $[34,43]$.

A motivation of this paper is to apply entanglement entropy to quantum field theory (QFT) with negative energy. We consider $d=4 \mathcal{N}=4$ SYM theory on $S^{1}$ [44]. Fermions become massive at tree level due to the antiperiodic boundary conditions along the compactified direction. Scalars and the $S^{1}$ component of the gauge field acquire a mass via quantum corrections. Thus, the IR behavior is dominated by that of $d=3$ pure Yang-Mills theory, a feature of which is confinement and a mass gap [45]. In terms of AdS/CFT, the IR region is cut off in the AdS soliton and the mass gap arises. Computing the mass of the AdS soliton using a standard method gives the negative answer. The mass at the conformal boundary of metrics asymptotic to the soliton is related via the AdS/CFT correspondence to the negative Casimir energy of a gauge theory on the boundary $S^{1} \times R^{1,2}$. The stability considerations imply that the soliton must be the lowest energy state with the given asymptotic conditions.

This negative energy arises due to the antiperiodic boundary condition on fermions, which breaks supersymmetry. Moreover, [8] shows that a twisted AdS soliton is dual to the Yang-Mills theory on $S^{1} \times R^{1,2}$ with twisted boundary conditions. The Casimir energy is also negative except for the extremal limit, where energy vanishes. Thus, Casimir energy depends on boundary conditions. It will be interesting to pursue it.

Another motivation is the computation of degrees of freedom from the entanglement entropy (e.g., the coefficient of the $A$-type anomaly for a spherical entangling surface in CFT $[46,47])$. One can define the renormalized entanglement entropy from the entanglement entropy with a spherical entangling surface [48]. It can give the central charge in CFT and show the number of the degrees of freedom. Since the central charge with the strip subsystem is not clear yet, however, it will be interesting to study it. The finite part of the entanglement entropy with the strip subsystem does not depend on the cutoff. It is proportional to the number of fields or the effective number of colors [e.g., $N^{2}$ of $d=4 \mathcal{N}=4 S U(N)$ SYM and $N^{3}$ of $d=6$ $(2,0) \mathrm{SCFT}[6]]$. The authors of [8] proposed the entropic $\mathrm{C}$ function from the entanglement entropy with the strip subsystem. The entropic $\mathrm{C}$ function does not depend on the cutoff and will measure degrees of freedom in general cases. The entropic $\mathrm{C}$ function decreases at large length in QFT dual to an AdS soliton and becomes a nice probe of the deconfinement phase transition.

In this paper, we switch on a background gauge field along an $S^{1}$ direction. A gauge transformation gives Wilson lines along $S^{1}$, which should change the boundary condition. Analyzing the gravity dual, we show that energy at the strong coupling increases with increase of the background gauge field and becomes even positive. Recall that the gauge field source is for the (conserved) current in the gauge/gravity correspondence. The vacuum expectation value (VEV) of the $S^{1}$ component of the current becomes nonzero $\left\langle J_{\phi}\right\rangle \neq 0$. This $\operatorname{VEV}\left\langle J_{\phi}\right\rangle$ also excites the state.

We analyze a phase transition as well as thermodynamic properties of the holographic entanglement entropy in a solitonic background with a background gauge field. We consider both small and large subregions of the entanglement entropy. We compute the entanglement entropy and the entropic $\mathrm{C}$ function. The latter becomes a nice probe of the confinement/deconfinement phase transition. We will demonstrate that a phase transition occurs for the KaluzaKlein mass (mass of a Kaluza-Klein state) $1 / l$ analogous to [8]. We also analyze the contribution of a background gauge field and the VEV $\left\langle J_{\phi}\right\rangle$ to the entropic C function [8]. We analyze the entanglement entropy at the small subregion. Computing the boundary energy and increasing amount of the entanglement entropy, we will obtain an entanglement temperature. As a by-product, we work out the generic formula for the second order correction to the holographic entanglement entropy with contributions from the deformation of the entangling surface. As a consistency check, we apply this formula to a spherical entangling surface and the resulting second order corrections are the same as ones presented in [35]. One can do a similar investigation in the strip case with some numerical simulation. We leave the problem to future work.

In Sec. II, we compute free energy of a QFT dual to a solitonic background with a background gauge field. In Sec. III, we compute the quasilocal stress tensor of the solitonic background. In Sec. IV, we compute the holographic entanglement entropy with a striped region. We analyze the confinement/deconfinement phase transition. We also introduce the entropic $\mathrm{C}$ function to probe a phase 
transition. For the small subregion, we compute the relation as in the first law of the thermodynamics. In Appendix A, we analyze the first law of HEE in higher dimension. In Appendix B, we compute the second order correction to the holographic entanglement entropy with spherical entanglement surface.

\section{FREE ENERGY}

In this section, we compute free energy of a QFT with a constant gauge field by using the gauge/gravity correspondence. The gravitational action with the Maxwell field has $U(1)$ gauge symmetry, which corresponds to $U(1)$ global symmetry in the field theory side. The action also includes the Gibbons-Howing boundary term to have a correct variation principle as follows [49]:

$$
\begin{aligned}
S= & \int d^{d+1} x \sqrt{-g}\left(\frac{1}{2 \kappa^{2}}\left(R+\frac{d(d-1)}{L^{2}}\right)-\frac{1}{4 g_{e}^{2}} F^{2}\right) \\
& +\frac{1}{2 \kappa^{2}} \int d^{d} x \sqrt{h}\left(2 K-\frac{2(d-1)}{L}\right),
\end{aligned}
$$

where $h$ is the induced metric at the AdS boundary, $K=h^{\mu \nu} \nabla_{\mu} n_{\nu}$, and a boundary term is added.

The Einstein equations of motion and the Maxwell equation become

$$
\begin{aligned}
R_{\mu \nu}-\frac{R}{2} g_{\mu \nu}-\frac{d(d-1)}{2 L^{2}} g_{\mu \nu} & =\frac{\kappa^{2}}{2 g_{e}^{2}}\left(2 F_{\mu \sigma} F_{\nu}{ }^{\sigma}-\frac{1}{2} g_{\mu \nu} F^{2}\right), \\
\nabla_{\mu} F^{\mu \nu} & =0 .
\end{aligned}
$$

The AdS soliton is a solution of equations of motion (EOM), which is the double Wick rotation of the AdS black hole. It describes the ground state of a QFT with the antiperiodic boundary condition on fermions. The following metric of an $\mathrm{AdS}_{d+1}$ soliton also becomes a solution of EOM as follows:

$d s_{d+1}^{2}=\frac{L^{2}}{z^{2}}\left(-d t^{2}+\frac{d z^{2}}{f_{d}(z)}+f_{d}(z) d \phi^{2}+\sum_{i=1}^{d-2} d x^{i} d x^{i}\right)$,

where

$f_{d}(z)=1-\left(1+\frac{\epsilon_{1} z_{+}^{2} a_{\phi}^{2}}{\gamma^{2}}\right)\left(\frac{z}{z_{+}}\right)^{d}+\frac{\epsilon_{1} z_{+}^{2} a_{\phi}^{2}}{\gamma^{2}}\left(\frac{z}{z_{+}}\right)^{2(d-1)}$,

where $a_{\phi}$ describes a constant gauge field and $\epsilon_{1}=-1{ }^{2}$ We have described $\gamma^{2}=\frac{(d-1) g_{e}^{2} L^{2}}{(d-2) \kappa^{2}}$, which is a dimensionless

\footnotetext{
${ }^{2}$ Note that the double Wick rotation $t=i \phi$ and $\phi=i t$ with $\epsilon_{1}=1$ corresponds to the Reissner-Nordström AdS black hole [49].
}

combination. The IR region $z>z_{+}$is cut off in (5). The background gauge field becomes

$$
A_{\phi}=a_{\phi}\left(1-\left(\frac{z}{z_{+}}\right)^{d-2}\right)
$$

where $A_{\phi}$ is regular at the tip of the soliton. The dual current is defined as $J^{\phi}=\frac{\delta S}{\delta a_{\phi}}$. The signature of $t$ does not affect the background gauge field unlike the Reissner-Nordström AdS black hole. Because a Wilson loop $\oint A$ vanishes around the vanishing circle at $z=z_{+}$, the gauge connection is regular at the tip of the soliton. The Kaluza-Klein mass of the $\phi$ circle is obtained in a Euclidean signature solution as follows:

$$
M_{0}=\frac{1}{4 \pi z_{+}}\left(d-\frac{\epsilon_{1}(d-2) z_{+}^{2} a_{\phi}^{2}}{\gamma^{2}}\right)>0 .
$$

Note that $M_{0}$ becomes nonzero for any real $a_{\phi}$ and the dimensionless ratio $a_{\phi} / M_{0}$ can smoothly be taken to be zero.

We have two branches solving the above equation in terms of $z_{+}$as follows:

$z_{+}=\frac{-2 \pi M_{0} \gamma^{2} \pm \sqrt{\left(2 \pi M_{0} \gamma^{2}\right)^{2}+d(d-2) \epsilon_{1} \gamma^{2} a_{\phi}^{2}}}{(d-2) \epsilon_{1} a_{\phi}^{2}}$.

The solution exists when $\left|a_{\phi}\right| \leq \frac{2 \pi M_{0} \gamma}{\sqrt{d(d-2)}}$. Choosing the minus sign in the above formula, $z_{+}$is divergent at the small $a_{\phi}$ limit. Since this background does not smoothly continue to the $\mathrm{AdS}_{d+1}$ soliton, it is not relevant for our analysis.

The free energy of the dual field theory is derived from analyzing the Euclidean action via an analytic continuation $Z=e^{-\beta F}=e^{-S_{E}\left(g_{*}\right)}$. The free energy becomes

$$
F=-\frac{L^{d-1}}{2 \kappa^{2} z_{+}^{d}}\left(1+\epsilon_{1} \frac{z_{+}^{2} a_{\phi}^{2}}{\gamma^{2}}\right) V_{d-1}
$$

Note that $L^{d-1} / \kappa^{2}$ is a dimensionless parameter and scales as in a power of $N$.

One can show that the solution of the plus sign is always stabler than that of the minus sign in (8) $(d \leq 9)$. We define a new parameter $a_{\phi}=\frac{2 \pi M_{0} \gamma}{\sqrt{d(d-2)}} x(|x| \leq 1)$. In $d=3$ and 4 , especially, the difference is computed as

$\frac{\kappa^{2}}{L^{d-1} M_{0}^{d}}\left(F_{-}-F_{+}\right)= \begin{cases}\frac{64}{27} \pi^{3}\left(1-x^{2}\right)^{\frac{3}{2}} \geq 0 & (d=3), \\ \pi^{4}\left(1-x^{2}\right)^{\frac{3}{2}} \geq 0 & (d=4) .\end{cases}$

Thus, we choose the plus sign in (8) in later study. 


\section{BOUNDARY STRESS TENSOR}

In this section we compute the stress tensor of boundary field theory dual to soliton background in two different ways. In the first method the Brown-York tensor with counterterms is used, and in the second the stress tensor is read from Fefferman-Graham (FG) expansion of metric near the boundary. Let us begin with the first way. The soliton metric (4) can be cast into the form (contemplate $d=4$ here)

$d s^{2}=F(z) d z^{2}+h_{a b}(z) d x^{a} d x^{b}, \quad x^{a}=t, \phi, x^{i}$

with

$$
h_{i j}=\frac{L^{2}}{z^{2}} \operatorname{diag}(-1, f(z), 1,1) .
$$

The boundary stress tensor near the boundary denoted by $\partial M$ (constant $z$ surface with $z \rightarrow 0$ ) is [50]

$$
T_{i j}=\left.\frac{1}{\kappa^{2}}\left(K_{i j}-K h_{i j}-\frac{3}{L} h_{i j}\right)\right|_{z \rightarrow 0},
$$

where $K_{i j}$ is the extrinsic curvature of the boundary $\partial M$. The first two terms are Brown-York tensor terms, and the last term is a counterterm added to yield a finite answer near the boundary. Substituting into the metric (4), the stress tensor can be derived. Let us focus on the $t t$ component which is relevant in the computation of boundary energy

$$
T_{t t}=-\frac{L \bar{a}_{\phi}}{2 \kappa^{2} z_{+}^{4}} z^{2}+O\left(z^{3}\right), \quad \bar{a}_{\phi}=1-\left(\frac{z_{+} a_{\phi}}{\gamma}\right)^{2} .
$$

It follows that the boundary energy is then [Eq. (12) in [50]]

$$
\begin{aligned}
M & =\int d^{2} x d \phi \sqrt{\sigma} u^{a} \xi^{b} T_{a b} \\
& =\int d^{2} x d \phi \sqrt{\sigma} u^{t} \xi^{t} T_{t t}=-\frac{V_{2}}{M_{0}} \frac{L^{3} \bar{a}_{\phi}}{2 \kappa^{2} z_{+}^{4}},
\end{aligned}
$$

where $\sigma_{i j}$ is the metric of a spacelike surface $\Sigma$ in $\partial M, u^{\mu}$ is the timelike unit vector normal to $\Sigma$. $\xi^{\mu}$ is a timelike Killing vector generating time translation isometry of the boundary. Here $\sqrt{\sigma}=\frac{\sqrt{f} L^{3}}{z^{3}}, u^{t}=\frac{z}{L}, \xi^{t}=1, \int d^{2} x=\int d x^{1} d x^{2}=V_{2}$. Note that the energy is negative when $a_{\phi}<a_{0}=\frac{2 \pi M_{0} \gamma}{d-1}$. When $a_{\phi}=0$, the above negative energy was compared with the negative Casimir energy of the gauge theory on $S^{1} \times R^{2}$. The result has a good agreement with [51]. When $a_{\phi}>a_{0}$, energy becomes even positive.

For metric (4) in general dimensions, we have

$$
h_{i j}=\frac{L^{2}}{z^{2}} \operatorname{diag}(-1, f(z), 1, \ldots, 1) .
$$

The $t t$ component of the quasilocal stress tensor becomes

$$
T_{t t}=-\frac{L \bar{a}_{\phi}}{2 \kappa^{2} z_{h}^{d}} z^{d-2}+O\left(z^{d-1}\right) .
$$

The boundary energy is computed as follows:

$$
M=\int d^{d-2} x d \phi \sqrt{\sigma} u^{t} \xi^{t} T_{t t}=-\frac{V_{d-2}}{M_{0}} \frac{L^{d-1} \bar{a}_{\phi}}{2 \kappa^{2} z_{+}^{d}},
$$

where $\sqrt{\sigma}=\frac{\sqrt{f} L^{d-1}}{z^{d-1}}, \int d^{2} x=V_{d-2}$. The energy in general dimensions is also negative when $a_{\phi}<a_{0}=\frac{2 \pi M_{0} \gamma}{d-1}$. The energy vanishes at $a_{\phi}=a_{0}$. When $a_{\phi}>a_{0}$, energy becomes positive.

Alternatively, we can obtain boundary stress tensor by resorting to FG expansion $[52,53]$. The bulk metric in FG gauge can be written as

$d s^{2}=\frac{L^{2}}{\tilde{z}^{2}}\left(d \tilde{z}^{2}+g_{\mu \nu} d x^{\mu} d x^{\nu}\right), \quad g_{\mu \nu}=\eta_{\mu \nu}+\delta g_{\mu \nu}$.

Here $\eta_{\mu \nu}$ is Minkowski flat metric, $\delta g_{\mu \nu}$ begins with terms of order $\tilde{z}^{d}$ near the boundary.

Next transforming the soliton background (4) into the form (19)

$$
\begin{aligned}
d s^{2} & =\frac{L^{2}}{z^{2}}\left(-d t^{2}+\frac{d z^{2}}{f_{d}(z)}+f_{d}(z) d \phi^{2}+\sum^{d-2} d x^{i} d x^{i}\right) \\
& =\frac{L^{2}}{\tilde{z}^{2}}\left(d \tilde{z}^{2}+g_{\mu \nu} d x^{\mu} d x^{\nu}\right)
\end{aligned}
$$

with asymptotic expansion

$$
\begin{aligned}
f_{d} & =1-\alpha z^{d}+\beta z^{2 d-2}, \quad \alpha=\frac{1}{z_{+}^{d}}\left(1-\frac{z_{+}^{2} a_{\phi}^{2}}{\gamma^{2}}\right), \\
\beta & =-\frac{1}{z_{+}^{2 d-4}} \frac{a_{\phi}^{2}}{\gamma^{2}},
\end{aligned}
$$

we obtain

$$
\begin{gathered}
g_{t t}=-\left(1+\alpha \frac{\tilde{z}^{d}}{d}+\beta \frac{\tilde{z}^{2 d-2}}{2(1-d)}\right), \\
g_{\phi \phi}=1+\beta \frac{(2 d-3) \tilde{z}^{2 d-2}}{2(d-1)}+\alpha\left(-1+\frac{1}{d}\right) \tilde{z}^{d}, \\
g_{i j}=\frac{\tilde{z}^{2}}{z^{2}}=\delta_{i j}\left(1+\alpha \frac{\tilde{z}^{d}}{d}+\beta \frac{\tilde{z}^{2 d-2}}{2(1-d)}\right) .
\end{gathered}
$$

The general variation of metric, to leading order, takes the form [35] 


$$
\delta g_{\mu \nu}=a \tilde{z}^{d} T_{\mu \nu}^{(0)}+\tilde{z}^{2 d-2}\left(b J_{\mu} J_{\nu}+c \eta_{\mu \nu} J^{2}\right),
$$

where $T^{(0)}$ is the boundary stress tensor, and the boundary current $J_{\mu}$ appears because the dual gauge field of the bulk

(4) is turned on. Here $a=\frac{2 \kappa^{2}}{d L^{d-1}}$ as given in [35], then from (25), we can read off the component of stress tensor:

$$
\begin{aligned}
T_{t t}^{(0)} & =-T_{x^{i} x^{i}}^{(0)}=-\frac{\alpha}{a d}=-\frac{L^{d-1}}{2 \kappa^{2}} \frac{1}{z_{+}^{d}}\left(1-\frac{z_{+}^{2} a_{\phi}^{2}}{\gamma^{2}}\right) \\
& =-\frac{L^{d-1}}{2 \kappa^{2}} \frac{1}{z_{+}^{d}} \bar{\alpha}_{\phi}, \\
T_{\phi \phi}^{(0)} & =\frac{\alpha}{a}\left(-1+\frac{1}{d}\right)=\frac{d L^{d-1}}{2 \kappa^{2}} \frac{1}{z_{+}^{d}}\left(1-\frac{z_{+}^{2} a_{\phi}^{2}}{\gamma^{2}}\right)\left(-1+\frac{1}{d}\right) .
\end{aligned}
$$

Note that (26) is energy density of boundary field theory which is consistent with (18).

\section{THE HOLOGRAPHIC ENTANGLEMENT ENTROPY}

We compute the holographic entanglement entropy [5,6] in the soliton background (4). We divide the boundary region into two regions. The first region is defined as $-l / 2 \leq x^{1} \leq l / 2$ and $0 \leq x^{i} \leq L_{x}$ for the remaining $x^{i}$, and wrapping $\phi$ direction. The second region is the complement. The boundary of the Ryu-Takayanagi surface ends on the boundary of the above region. The surface becomes a codimension 2 surface at a constant time slice with the embedding scalar $z=z\left(x^{1}\right)$. The surface action becomes

$$
A=L_{x}^{d-2} \int d x^{1}\left(\frac{L}{z}\right)^{d-1} \sqrt{\left(z^{\prime}\right)^{2}+f_{d}(z)}
$$

The Hamiltonian of $A$ becomes a constant independent of $x_{1}$. It leads to the following EOM of the first order,

$$
z^{\prime}=\sqrt{f_{d}(z)\left(\frac{f_{d}(z)}{f_{d}\left(z_{*}\right)} \frac{z_{*}^{2(d-1)}}{z^{2(d-1)}}-1\right)},
$$

where $z_{*}$ is the turning point. $z^{\prime}=0$ at $z=z_{*}$. By integrating $z^{\prime}$, we require the boundary condition

$$
l=2 \int_{\epsilon}^{z_{*}} d z \frac{1}{\sqrt{f_{d}\left(\frac{f_{d}(z) z_{*}^{2(d-1)}}{f_{d}\left(z_{*}\right) z^{2(d-1)}}-1\right)}} .
$$

The above formula relates $l$ with $z_{*}$. Substituting (29), the surface action (28) becomes

$$
A=2 L_{x}^{d-2} \int_{\epsilon}^{z_{*}} d z \frac{L^{d-1} z^{2-2 d}}{\sqrt{z^{2-2 d}-\frac{f_{d}\left(z_{*}\right)}{f_{d}(z)} z_{*}^{2-2 d}}},
$$

where $\epsilon$ is a small cutoff scale. The singular part of $A$ becomes $A \sim \frac{2 L_{x}^{d-2} L^{d-1}}{(d-2) \epsilon^{d-2}}$.

For pure AdS, the surface action (31) can be integrated over a region. Replacing $z_{*}$ with $l$, it becomes

$$
\begin{aligned}
A= & 2 L_{x}^{d-2} L^{d-1}\left(\frac{1}{(d-2) \epsilon^{d-2}}\right. \\
& \left.-\frac{2^{d-2} \pi^{d-1}}{d-2}\left(\frac{\Gamma\left(\frac{d}{2(d-1)}\right)}{\Gamma\left(\frac{1}{2(d-1)}\right)}\right)^{d-1} \frac{1}{l^{d-2}}\right) .
\end{aligned}
$$

\section{A. The confinement/deconfinement transition}

According to $[8,9]$, the holographic entanglement entropy can capture the confinement/deconfinement phase transition without black brane solutions. In this section, we analyze the confinement/deconfinement transition applying it to a solitonic background with a background gauge field along the $\phi$ direction. The entanglement entropy counts the degrees of freedom of the entangled states at the energy scale $\Lambda \sim 1 / l$. When $l$ is large, it probes the IR limit.

We have two choices of the minimal surface. The first one is a connected surface (31), which corresponds to the deconfinement phase. The second one is a disconnected surface, which goes straight from the AdS-soliton boundary to the bulk. Because the disconnected surface does not depend on $l$, it corresponds to the confinement phase.

The connected surface (31) has the maximal size $l_{\max }$ of the interval, which depends on the background gauge field $a_{\phi}$ and the Kaluza-Klein mass $M_{0}$. The size $l M_{0}$ of the interval is plotted as a function of $z_{*}$ in three dimensions in Fig. 1 . The size $l M_{0}$ has the maximal value $l_{\max } M_{0} \sim 1$. Explicitly, $l_{\max } M_{0}=0.39$ when $a_{\phi}=a_{c}\left(=2 \pi M_{0} \gamma /\right.$ $\sqrt{d(d-2)})$. This is larger than that of the $\mathrm{AdS}_{5}$ soliton. When $a_{\phi}$ becomes imaginary, the curve $a_{\phi}=5 i \gamma$ leads to the result of the geometric entropy [54]. The geometric entropy is related to the entanglement entropy via the double Wick rotation $[55,56]$.

When $l$ becomes large, the connected surface does not exist anymore. Instead, the disconnected surface dominates the behavior. It ends at the tip of the soliton $\left(z=z_{+}\right)$:

$$
\begin{aligned}
A^{\mathrm{dis}} & =2 L_{x}^{d-2} L^{d-1} \int_{\epsilon}^{z_{+}} d z \frac{1}{z^{d-1}} \\
& =\frac{2 L_{x}^{d-2} L^{d-1}}{d-2}\left(\frac{1}{\epsilon^{d-2}}-\frac{1}{z_{+}^{d-2}}\right) .
\end{aligned}
$$

The difference $\Delta A=A-A^{\text {dis }}$ in three dimensions is plotted in Fig. 2. There are two connected surfaces of the same curve. The larger one is unphysical because the lower 


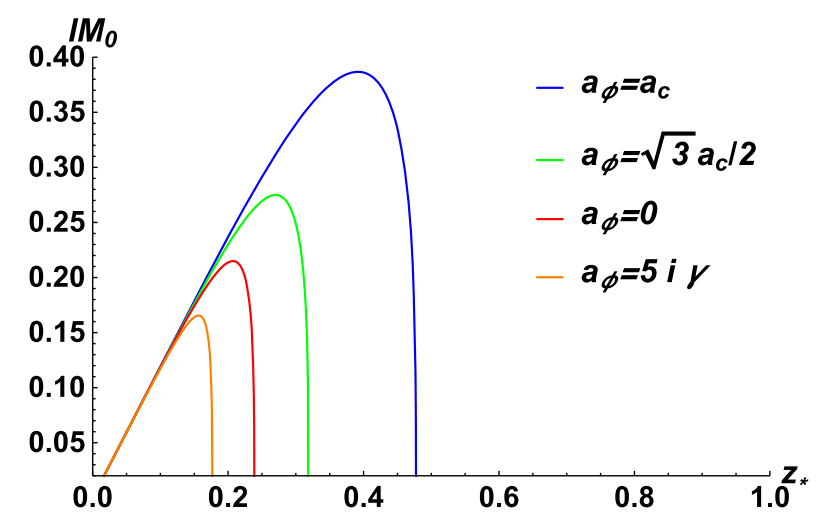

FIG. 1. The size $l M_{0}$ of the subregion with some fixed $a_{\phi}$ as a function of $z_{*}$ in $d=3$. At the special value $a_{\phi}=a_{0}$, the boundary energy is zero. In the figure $a_{0}=\sqrt{3} a_{c} / 2$ $\left[a_{c}=2 \pi M_{0} \gamma / \sqrt{d(d-2)}\right]$. The size $l$ linearly grows for small $z_{*}$ and has the maximal value $l_{\max }=0.39,0.27,0.22,0.17$ in units of $1 / M_{0}$ from the top to the bottom, respectively. The figure shows that $z_{*}$ has two values.

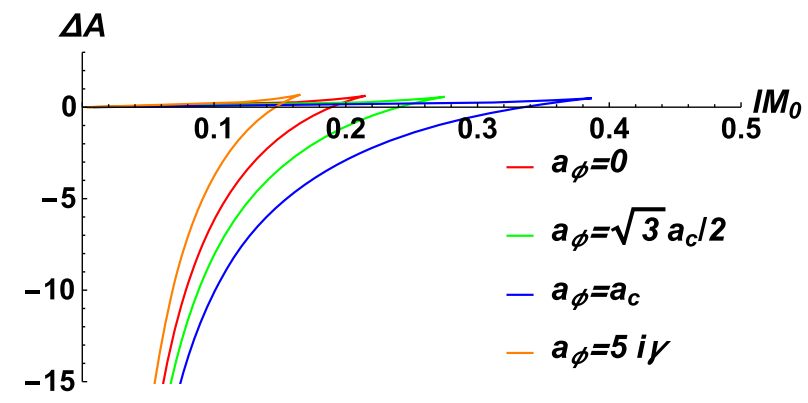

FIG. 2. The entanglement entropy as a function of $l$ in three dimensions. The larger surface is unphysical between two connected surfaces of the same curve. When $0<l<l_{c}$, the connected surface is favored. When $l>l_{c}$, the disconnected surface dominates the behavior. The critical length is given by $l_{c}=0.34,0.24,0.19,0.15$ in units of $1 / M_{0}$ from the bottom to the top, respectively. This implies that the phase transition occurs at the large Kaluza-Klein mass $M_{0}$ with increase of the background gauge field $a_{\phi}$.

entropy is a dominant contribution to the path integral. For large $l$, the disconnected surface dominates the behavior. There is a first order phase transition at a critical point $l=l_{c}$. The critical length increases with increase of $a_{\phi}$ in general.

Because two surfaces correspond to confinement and deconfinement phases, this phase transition can be interpreted as the confinement/deconfinement transition. The disconnected surface (33) corresponds to the confinement phase. Because the disconnected surface does not depend on the size $l$, there is no correlation at a large distance due to the mass gap $M_{0}$.

To probe the confinement/deconfinement phase transition, we introduce the entropic $\mathrm{C}$ function. It was proposed in [8]. The entropic $\mathrm{C}$ function is defined as

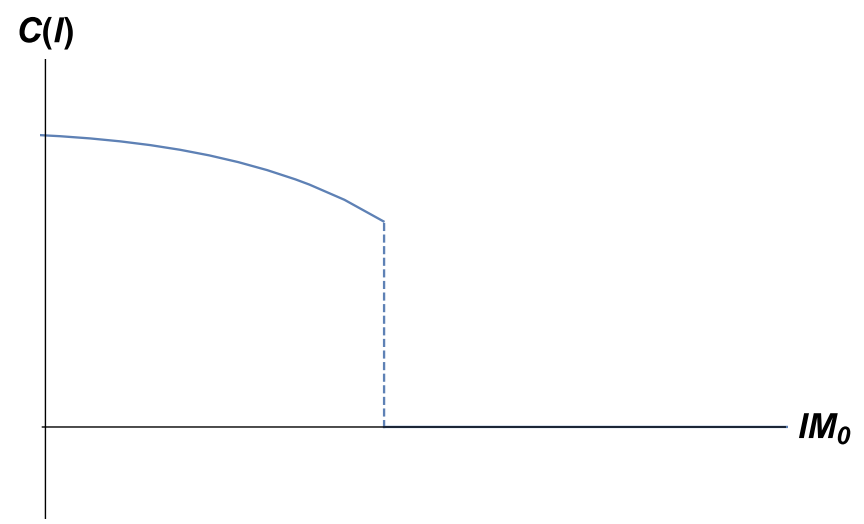

FIG. 3. The entropic $\mathrm{C}$ function as a function of $l M_{0}$ at small $a_{\phi}$. $C(l)$ decreases with increase of $l M_{0}$. At the critical point $l=l_{c}$, it jumps and becomes zero. This figure explains the confinement/deconfinement phase transition. Finally, this figure implies that degrees of freedom (d.o.f.) of entangling states are frozen in the confined phase.

$$
C(l)=\frac{l^{d-1}}{V} \frac{d S}{d l}
$$

where $V=L_{x}^{d-2}$. This is the generalization of the twodimensional entropic $C$ function defined in $[57,58]$. The entropic $\mathrm{C}$ function shows degrees of freedom at an energy scale $1 / l$. For the pure AdS, the $\mathrm{C}$ function becomes

$$
C(l)=C_{0} \equiv \frac{L^{d-1} 2^{d} \pi^{\frac{d+1}{2}}}{\kappa^{2}}\left(\frac{\Gamma\left(\frac{d}{2(d-1)}\right)}{\Gamma\left(\frac{1}{2(d-1)}\right)}\right)^{d-1} .
$$

The entropic $\mathrm{C}$ function is plotted as a function of $l M_{0}$ in Figs. 3 and 4. When $a_{\phi}$ is small or an imaginary number, the entropic $\mathrm{C}$ function decreases with increase of $l M_{0}$. The entropic $\mathrm{C}$ function suddenly becomes 0 at the critical point $l=l_{c}$. Figure 3 implies that there are no d.o.f. in the confined phase. In Fig. 4 (left), the entropic C function increases until the middle of the horizontal line. After passing the peak, it decreases. Figure 4 (right) shows a competitive behavior between $l^{d-1}$ and $\partial S / \partial l$. The product of these becomes $C(l)$, which becomes nonmonotonic. This figure also shows $S^{\prime \prime}(l) \leq 0$ (concave $S$ ), which agrees with the strong subadditivity applied to the entanglement entropy $[59,60]$.

There is a characteristic length of the system $1 / M_{0}$ which is the radius of the spatial cycle denoted by $S^{1}$ in the soliton background; the entanglement entropy counts the degrees of freedom (d.o.f.) of the entangled states at the energy scale of the subsystem size $l(E \sim 1 / l)$ between the subsystem and the complement. Once the subsystem size $l$ chosen here is smaller than the characteristic length $\left(E \gtrsim M_{0}\right)$, the entanglement entropy can detect the effective degree of freedom encoding on the $S^{1}$. While these d.o.f. cannot be detected by entanglement entropy 

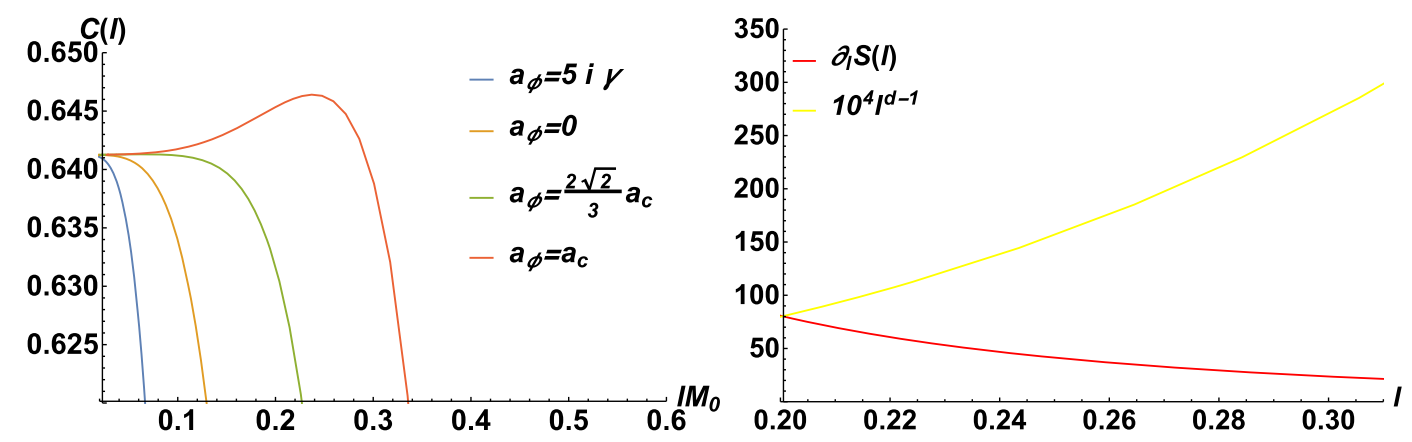

FIG. 4. Left: the entropic C function as a function of $l M_{0}$ with some fixed $a_{\phi} . C(l)$ can increase until the middle when $a_{\phi}=a_{c}$. It decreases after passing the peak. The green line is a critical line due to the analytical calculation (45). Lines above the green line increase at first, and the line below the green line decreases. Right: the competition between $l^{d-1}$ and $\partial S / \partial l$ terms when $a_{\phi}=a_{c}$ and $M_{0}=1$. Compared with the left figure, this explains a nonmonotonic behavior of $C(l)$.

once the subsystem size is much larger than the characteristic length $\left(E \ll M_{0}\right)$, the d.o.f. will be smeared and the entanglement entropy cannot identify such kinds of d.o.f. hidden in $S^{1}$. In this sense, then entanglement $\mathrm{C}$ function in the small subsystem can monotonically increase vs the length of the subsystem. However, once the subsystem size is comparable with the characteristic length of the system $\left(E \sim M_{0}\right)$, the d.o.f. cannot be detectable which leads to the entropy $\mathrm{C}$ function monotonically decreasing with respect to the size of the subsystem. That is a one interpretation of the nonmonotonic behavior of entropy $\mathrm{C}$ function with respect to subsystem size. The other interpretation of the nonmonotonic behavior of the entropy $\mathrm{C}$ function is that the Lorentz symmetry on the boundary of the AdS soliton background has been explicitly broken [58]. In this sense, the monotonic behavior of the entropy $\mathrm{C}$ function cannot be protected. Here we generalize the entropy $\mathrm{C}$ function for the spherical entanglement surface to the one for strip case. In terms of $[48,61]$, the monotonic behavior of entropy C function for the spherical entanglement surface can be valid. In general, the entropy $\mathrm{C}$ function depends on the shape of the entanglement surface. One can express the entropy $\mathrm{C}$ function $C_{4}^{(\Sigma)}$ as follows:

$$
C_{4}^{(\Sigma)}=2 a_{4} \int_{\Sigma} d^{2} \sigma \sqrt{h} E_{2}+c_{4} \int_{\Sigma} d^{2} \sigma \sqrt{h} I_{2},
$$

where the $\Sigma$ is entanglement surface, $a_{4}, c_{4}$ are associated with the four-dimensional a type and c type anomaly respectively, and $E_{2}$ is Euler density and $I_{2}$ is built from the Weyl tensor. In particular, we have studied the strip entanglement surface and the entropy C function may not fully correspond to the central charge of the boundary field theory, since the entropy C function for the strip contains the two types of anomalies, such as a type and c type anomalies. In this sense, the entropy $\mathrm{C}$ function in our case is the combination of a and c. For the spherical case, the $I_{2}$ is vanishing. That means the spherical case is a good choice to test the monotonical behavior of the entropy $\mathrm{C}$ function. Due to this reason, one cannot make sure whether the central charge behaves indeed monotonically with respect to the variation of the scale. We would like to investigate this issue in the near future.

\section{B. HEE in a small region $(d=3)$}

Since we are interested in a small subregion, we expand the action and $l$ in terms of small $z_{*}$. Near the AdS-soliton boundary neglecting the information of the infrared region, $z_{*} \ll z_{+}$. The leading order contribution of the surface comes from the AdS boundary and is the zero temperature entanglement entropy in the infinite volume. Since the background gauge field and the Kaluza-Klein (KK) mass (or finite volume corrections) are small, one can use the perturbation in terms of $z_{*}: M_{0} z_{*} \sim M_{0} l \ll 1$ and $a_{\phi} l \ll 1$.

We compute small deviations in $d=3$. The size of the interval $l$ is expanded in a power series as follows:

$$
\begin{aligned}
l= & 2 z_{*}(E(-1)-K(-1))+\frac{\bar{a}_{\phi} z_{*}^{4}\left(\frac{1}{2} E(-1)-\frac{1}{2} K(-1)-\frac{\pi}{8}\right)}{z_{+}^{3}}+\frac{2\left(\bar{a}_{\phi}-1\right) z_{*}^{5}(E(-1)-K(-1))}{5 z_{+}^{4}} \\
& +\frac{\bar{a}_{\phi}^{2} z_{*}^{7}\left(\frac{5}{16} E(-1)-\frac{5}{21} K(-1)-\frac{\pi}{8}\right)}{z_{+}^{6}}+\frac{\left(\bar{a}_{\phi}-1\right) \bar{a}_{\phi} z_{*}^{8}\left(\frac{1}{2}(E(-1)-K(-1))-\frac{1}{64} \pi\right)}{z_{+}^{7}} \cdots,
\end{aligned}
$$


where $\bar{a}_{\phi}=1+\epsilon\left(\frac{z_{+} a_{\phi}}{\gamma}\right)^{2} . E(k)$ and $K(k)$ are elliptic integrals. The action is expanded in a power series as follows:

$$
\begin{aligned}
\frac{A}{L_{x} L^{2}}= & \frac{2}{\epsilon}+\frac{2(K(-1)-E(-1))}{z_{*}}+\frac{\bar{a}_{\phi} z_{*}^{2}\left(\frac{1}{2} E(-1)-\frac{1}{2} K(-1)-\frac{\pi}{4}\right)}{z_{+}^{3}}+\frac{z_{*}^{3}\left(\bar{a}_{\phi}-1\right)(E(-1)-K(-1))}{z_{+}^{4}} \\
& +\frac{\bar{a}_{\phi}^{2} z_{*}^{5}\left(\frac{3}{16} E(-1)-\frac{1}{12} K(-1)-\frac{\pi}{8}\right)}{z_{+}^{6}}+\frac{z_{*}^{6}\left(\bar{a}_{\phi}-1\right) \bar{a}_{\phi}(12 E(-1)-12 K(-1)-\pi)}{16 z_{+}^{7}} \\
& -\frac{3\left(\bar{a}_{\phi}-1\right)^{2} z_{*}^{7}(E(-1)-K(-1))}{20 z_{+}^{8}}+\frac{\bar{a}_{\phi}^{3} z_{*}^{8}\left(\frac{7}{64} E(-1)+\frac{1}{48} K(-1)-\frac{3 \pi}{32}\right)}{z_{+}^{9}}+\cdots
\end{aligned}
$$

Note that $z_{+}=\frac{d}{4 \pi M_{0}}+O\left(a_{\phi}^{2}\right)$ when $a_{\phi} \ll M_{0}$. The above expansion is in terms of the KK mass when $a_{\phi}=0$. For nonzero $a_{\phi}$, the above result also shows corrections of background gauge fields.

HEE is written up to order $l^{5}$ as follows:

$$
\begin{aligned}
\frac{\kappa^{2}}{2 \pi} S= & A=\frac{2 L_{x} L^{2}}{\epsilon}-\frac{8 \pi^{3} L_{x} L^{2}}{\Gamma\left(\frac{1}{4}\right)^{4} l} \\
& +L_{x} L^{2}\left(-\frac{4 \Gamma\left(\frac{5}{4}\right)^{4} \bar{a}_{\phi} l^{2}}{\pi^{2} z_{+}^{3}}+\frac{3 \Gamma\left(\frac{1}{4}\right)^{4}\left(\bar{a}_{\phi}-1\right) l^{3}}{80 \pi^{3} z_{+}^{4}}\right) \\
& +O\left(l^{5}\right) .
\end{aligned}
$$

For very small $l$, the increased amount of HEE becomes

$$
\Delta S=-\frac{8 L_{x} L^{2} \Gamma\left(\frac{5}{4}\right)^{4} \bar{a}_{\phi} l^{2}}{\kappa^{2} \pi z_{+}^{3}}
$$

where $\Delta S$ becomes negative when $a_{\phi}<\frac{2 \pi M_{0} \gamma}{d-1}$. Because quarks cannot be isolated in confinement, confinement decreases degrees of freedom. The $O\left(l^{3}\right)$ contribution coming from the real gauge field $a_{\phi}$ is also negative and similar to HEE of the Reissner-Nordström AdS black hole [40].

The energy difference in the dual field theory is defined as

$$
\Delta E=T_{t t}^{(0)} L_{x} l=-\frac{L_{x} l L^{2} \bar{a}_{\phi}}{2 \kappa^{2} z_{+}^{3}},
$$

where $T_{t t}^{(0)}$ is defined in (26) and $\Delta E$ is proportional to the small volume $l L_{x}=l / M_{0}$.

The relation like the first law of thermodynamics is as follows:

$$
\Delta E=T_{\text {ent }} \Delta S,
$$

where entanglement temperature $[22,24]$ is defined as

$$
T_{\mathrm{ent}}=\frac{\pi}{16 \Gamma\left(\frac{5}{4}\right)^{4} l}
$$

Entanglement temperature is inversely proportional to the length $l$. Its coefficient is known to be universal in asymptotic AdS black holes $\Delta S=\Delta E /\left(2 T_{\text {ent }}\right)$ [62]. However, it is different from $\operatorname{AdS}$ solitons by a factor because the increased amount of HEE $\Delta S$ generally includes $T_{x^{i} x^{i}}$ in a striped subregion [35]: An AdS soliton $\left(a_{\phi}=0\right)$ gives negative energy density of boundary field theory $T_{t t}^{(0)}=T_{i}^{(0) i}=-T_{x^{i} x^{i}}^{(0)}<0$ unlike AdS black holes. The increased amount of HEE can be computed from Eq. (3.78) of [35]. Note that the expansion (37) and (38) can be computed until higher orders. HEE becomes of $O\left(\bar{a}_{\phi}^{2} l^{5} / z_{+}^{6}\right)$ at the next order.

In Appendix A, we computed entanglement temperature in other dimensions $(d \geq 5)$. The increased amount of HEE become negative as in the energy difference when $a_{\phi}<\frac{2 \pi M_{0} \gamma}{d-1}$. That is, confinement decreases degrees of freedom.

Our results including three-dimensional ones $(d \geq 3)$ are summarized as follows:

$$
\frac{\Delta E}{\Delta S} \equiv T_{\text {ent }}=\frac{(d+1) \Gamma\left(\frac{d+1}{2 d-2}\right) \Gamma\left(\frac{3 d-2}{2 d-2}\right)^{2}}{\sqrt{\pi} d^{2} \Gamma\left(\frac{d}{d-1}\right) \Gamma\left(\frac{2 d-1}{2 d-2}\right)^{2}} \frac{1}{l} .
$$

Entanglement temperature is different from that of AdS black holes by a factor 2 again. The above formula shows that the amount of information inside an interval $l$ is proportional to the energy inside the region surrounded by the entangling surface.

We also evaluate the entropic $\mathrm{C}$ function for small $l$. The entropic $\mathrm{C}$ function is sensitive to the d.o.f. at the energy scale $E \sim 1 / l$. Making use of (34), it becomes

$$
C(l)=C_{0}+\frac{2 T_{t t}^{(0)} l^{d-1}}{T_{\mathrm{ent}}}+O\left(q l^{d}\right) .
$$

The energy density $T_{t t}^{(0)}$ is negative when $a_{\phi}<\frac{2 \pi M_{0} \gamma}{d-1}$. Moreover, $C(l)$ decreases with increase of $l\left(M_{0} l \lesssim 1\right)$. On the other hand, the energy density $T_{t t}^{(0)}$ becomes positive when $a_{\phi}>\frac{2 \pi M_{0} \gamma}{d-1}$ : a constant gauge field is of the same order as the KK mass $\left(a_{\phi} l / \gamma \sim M_{0} l \lesssim 1\right)$. The entropic C 
function $C(l)$ increases with increase of $l$. Here it is not necessary that the c-theorem $c^{\prime}(l) \leq 0$ has monotonic behavior in a theory with breaking Lorentz symmetry explicitly. Note that results (44) and (45) do not apply to a three-dimensional soliton with a background gauge field.

\section{DISCUSSION}

We analyzed the confinement/deconfinement phase transition and thermodynamic properties of the holographic entanglement entropy in a soliton background with a background gauge field. The phase transition occurs at the scale $l M_{0} \sim 1$ due to the competition of two minimal surfaces as analogous to [8] (see Fig. 2). Our result implies that the dual QFT is a confining theory and there is no correlation at a large distance due to the mass gap and confinement.

We also computed the entropic $\mathrm{C}$ function $C(l)$, which probed a phase transition. The entropic $\mathrm{C}$ function for small $a_{\phi}$ decreases with increase of $l$ as usual for the Zamolodchikov $\mathrm{C}$ function in two-dimensional theories. However, a large amount of $a_{\phi}$ makes behaviors nontrivial. When $E \gtrsim M_{0}$, HEE can detect the effective degrees of freedom of entangling states inside the circle. When $a_{\phi}>\frac{2 \pi M_{0} \gamma}{d-1}, C(l)$ increases with increase of $l$ and does not comply with the c-theorem. When $E \lesssim M_{0}$, it cannot detect effective degrees of freedom inside the circle and decrease. Although $C(l)$ is nonmonotonic, it still always satisfies the condition $C(l \rightarrow 0) \geq C(l \rightarrow \infty)$. Note that the two-dimensional entropic $\mathrm{C}$ function $C_{d=2}(=l d S / d l)$ satisfies $d C_{d=2} / d l \leq 0$ by applying the strong subadditivity to quantum field theories $[57,58]$. However, the coefficient is $l^{d-1}$ instead of $l$ in the definition of the general entropic $\mathrm{C}$ function $C(l)$ $(d \neq 2)$ and makes $C(l)$ independent of $l$ at small $l$. Thus, it does not need to satisfy the analogous condition. See also the nonmonotonic behaviors of renormalized entanglement entropy in the $4 d$ example such as the steep domain wall [48] (see also [63]).

We also pointed out the two possible reasons to understand the nonmonotonic behavior of entropic $\mathrm{C}$ function for the strip case. The first one is entanglement entropy can count the effective degrees of freedom up to some specific energy scale. The other is entropic C function depends on the shape of the entanglement surface and the entropic $\mathrm{C}$ function contains a type and c type anomaly contributions respectively. It is interesting to study their effects on the entropic C function in the future.

We derived the relation as in the first law of thermodynamics. The entanglement temperature is defined in (44) and becomes an inverse function of $l$. We find that both the boundary energy and the increased amount of HEE become negative in the field theory side when $a_{\phi}<\frac{2 \pi M_{0} \gamma}{d-1}$. That is, confinement decreases degrees of freedom. On the other hand, $a_{\phi}$ can increase degrees of freedom and makes both quantities positive. As seen in (44) and (45), the sign of the increased amount of HEE or the energy density affects nonmonotonic behavior of the entropic $\mathrm{C}$ function.

In Appendix B, the generic formula for the second order correction to the holographic entanglement entropy with contributions from the deformation of the entangling surface has been given. We apply this formula to a spherical entangling surface and reproduce the resulting second order corrections the same as ones given in [35].

We showed that the AdS soliton with the background gauge field corresponded to a QFT with constant gauge fields (Wilson lines). This QFT is nontrivial because it also has the nonzero VEV $\left\langle J_{\phi}\right\rangle$. So, it will be interesting to analyze dependence of a constant gauge field in other physics such as the confinement/deconfinement phase transition between an AdS soliton and the AdS black hole.

Moreover, the comparison between the field theory and the gravity dual will be possible in two dimensions. A two-dimensional spin $\frac{1}{2}$ field with a background gauge field on the cylinder can change signs of vacuum energy. The periodic spin $\frac{1}{2}$ field with flat gauge connection has an equivalent description in terms of twisted boundary conditions. That is, a constant gauge field along the $S^{1}$ direction can be changed into a twisted boundary condition: $\psi(t \cdot x+L)=e^{-2 \pi i \nu} \psi(t, x)$ via a gauge transformation, where a parameter $\nu$ is related with Wilson lines (see [64]). Vacuum energy of a spin $\frac{1}{2}$ field satisfying a twisted boundary condition becomes [65]

$$
E_{F 0}=-4 E_{0}
$$

where $E_{0}$ becomes vacuum energy of a scalar as follows:

$$
E_{0}=\frac{2 \pi}{L} \sum_{n=1}^{\infty}(n-\nu)=\frac{2 \pi}{L}\left(\frac{1}{24}-\frac{1}{8}(2 \nu-1)^{2}\right) .
$$

Here, we have introduced a cutoff factor $e^{-(n-\nu) \alpha / L}$ into the divergent sums in the second equality $(\alpha \rightarrow 0)$ and have discarded a cutoff dependent term. For the periodic and antiperiodic boundary conditions, $E_{F 0}=\frac{2 \pi}{3 L}(\nu=0)$ and $E_{F 0}=-\frac{\pi}{3 L}\left(\nu=\frac{1}{2}\right)$, respectively. Thus, the constant $\nu$ can simultaneously change the boundary condition and vacuum energy. The entanglement entropy of spin $\frac{1}{2}$ theories should be comparable with those of an $\mathrm{AdS}_{3}$ soliton with a background gauge field. However, one needs to take care of the mass gap, which is taken into account in [66]. 


\section{ACKNOWLEDGMENTS}

M. F. would like to thank B. S. Kim and T. Takayanagi for useful discussions related to this work. M. F. is supported by the Natural Science Foundation of China under the Grant No. 11850410431. S. H. would like to appreciate the financial support from Jilin University and Max Planck Partner group, as well as Natural Science Foundation of China Grants (No. 12075101). Y. S. would like to thank the support from China Postdoctoral Science Foundation (No. 2019M653137).

\section{APPENDIX A: THE FIRST LAW IN FIVE- AND SIX-DIMENSIONAL QFTS}

In this section, we analyze the deviation from the infinite volume and $d=5 . l$ and the action are expanded in a power series as follows:

$$
\begin{aligned}
l= & \frac{2 \sqrt{\pi} \Gamma\left(\frac{13}{8}\right) z_{*}}{5 \Gamma\left(\frac{9}{8}\right)}+\frac{\bar{a}_{\phi} z_{*}^{6}}{z_{+}^{5}}\left(\frac{\sqrt{\pi} \Gamma\left(\frac{13}{8}\right)}{20 \Gamma\left(\frac{9}{8}\right)}-\frac{\sqrt{\pi} \Gamma\left(\frac{5}{4}\right)}{12 \Gamma\left(\frac{3}{4}\right)}\right)+\frac{4 \sqrt{\pi} \Gamma\left(\frac{13}{8}\right)\left(\bar{a}_{\phi}-1\right) z_{*}^{9}}{45 \Gamma\left(\frac{9}{8}\right) z_{+}^{8}}+\frac{z_{*}^{11} \bar{a}_{\phi}^{2}}{z_{+}^{10}}\left(-\frac{\sqrt{\pi} \Gamma\left(\frac{5}{4}\right)}{16 \Gamma\left(\frac{3}{4}\right)}+\frac{9 \sqrt{\pi} \Gamma\left(\frac{13}{8}\right)}{320 \Gamma\left(\frac{9}{8}\right)}-\frac{7 \sqrt{\pi} \Gamma\left(\frac{15}{8}\right)}{704 \Gamma\left(\frac{11}{8}\right)}\right) \\
& +\frac{z_{*}^{14} \bar{a}_{\phi}\left(\bar{a}_{\phi}-1\right)}{z_{+}^{13}}\left(-\frac{\sqrt{\pi} \Gamma\left(\frac{5}{4}\right)}{84 \Gamma\left(\frac{3}{4}\right)}+\frac{\sqrt{\pi} \Gamma\left(\frac{13}{8}\right)}{10 \Gamma\left(\frac{9}{8}\right)}\right) \cdots,
\end{aligned}
$$

and

$$
\begin{aligned}
\frac{A}{L_{x}^{3} L^{4}}= & \frac{2}{3 \epsilon^{3}}-\frac{2 \sqrt{\pi} \Gamma\left(\frac{13}{8}\right)}{15 \Gamma\left(\frac{9}{8}\right) z_{*}^{3}}+\left(\frac{\sqrt{\pi} \Gamma\left(\frac{13}{8}\right)}{20 \Gamma\left(\frac{9}{8}\right)}-\frac{\sqrt{\pi} \Gamma\left(\frac{5}{4}\right)}{4 \Gamma\left(\frac{3}{4}\right)}\right) \frac{z_{*}^{2} \bar{a}_{\phi}}{z_{+}^{5}}+\frac{\sqrt{\pi} \Gamma\left(\frac{13}{8}\right)}{5 \Gamma\left(\frac{9}{8}\right)} \frac{z_{*}^{5}\left(\bar{a}_{\phi}-1\right)}{z_{+}^{8}} \\
& +\left(\frac{\sqrt{\pi} \Gamma\left(\frac{13}{8}\right)}{64 \Gamma\left(\frac{9}{8}\right)}-\frac{\sqrt{\pi} \Gamma\left(\frac{5}{4}\right)}{16 \Gamma\left(\frac{3}{4}\right)}-\frac{\sqrt{\pi} \Gamma\left(\frac{15}{8}\right)}{64 \Gamma\left(\frac{11}{8}\right)}\right) \frac{z_{*}^{7} \bar{a}_{\phi}^{2}}{z_{+}^{10}} \cdots
\end{aligned}
$$

The above expansion is able to be used to compute corrections in terms of Kaluza-Klein mass and the background gauge field.

HEE is rewritten up to order $l^{5}$ as follows:

$$
\frac{\kappa^{2}}{2 \pi} S=A=\frac{2 L_{x}^{3} L^{4}}{3 \epsilon^{3}}-\frac{16 \pi^{2} \Gamma\left(\frac{13}{8}\right)^{4} L_{x}^{3} L^{4}}{1875 \Gamma\left(\frac{9}{8}\right)^{4} l^{3}}+L_{x}^{3} L^{4}\left(-\frac{25 \Gamma\left(\frac{9}{8}\right)^{2} \Gamma\left(\frac{5}{4}\right) \bar{a}_{\phi} l^{2}}{24 \sqrt{\pi} \Gamma\left(\frac{3}{4}\right) \Gamma\left(\frac{13}{8}\right)^{2} z_{+}^{5}}+\frac{3125 \Gamma\left(\frac{9}{8}\right)^{4}\left(\bar{a}_{\phi}-1\right) l^{5}}{288 \pi^{2} \Gamma\left(\frac{13}{8}\right)^{4} z_{+}^{8}}\right) \cdots
$$

The increased amount of HEE becomes

$$
\Delta S=-\frac{25 \sqrt{\pi} \Gamma\left(\frac{9}{8}\right)^{2} \Gamma\left(\frac{5}{4}\right) \bar{a}_{\phi} l^{2} L_{x}^{3} L^{4}}{12 \Gamma\left(\frac{3}{4}\right) \Gamma\left(\frac{13}{8}\right)^{2} \kappa^{2} z_{+}^{5}}
$$

$\Delta S$ becomes negative when $a_{\phi}<\frac{2 \pi M_{0} \gamma}{d-1}$. Because quarks cannot be isolated in confinement, confinement decreases degrees of freedom of entangled states.

The increased amount of energy in the dual field theory is defined as

$$
\Delta E=-\frac{l L_{x}^{3} L^{4} \bar{a}_{\phi}}{2 \kappa^{2} z_{+}^{5}}
$$

Using the relation like the first law $\Delta E=T_{\text {ent }} \Delta S$, entanglement temperature is defined as

$$
T_{\mathrm{ent}}=\frac{6 \Gamma\left(\frac{3}{4}\right) \Gamma\left(\frac{13}{8}\right)^{2}}{25 \sqrt{\pi} \Gamma\left(\frac{9}{8}\right)^{2} \Gamma\left(\frac{5}{4}\right) l}
$$


We analyze the deviation in six dimensions. The size $l$ and the action are expanded in a power series as follows:

$l=\frac{\sqrt{\pi} \Gamma\left(\frac{8}{5}\right) z_{*}}{3 \Gamma\left(\frac{11}{10}\right)}+\left(\frac{\sqrt{\pi} \Gamma\left(\frac{8}{5}\right)}{30 \Gamma\left(\frac{11}{10}\right)}-\frac{2 \sqrt{\pi} \Gamma\left(\frac{6}{5}\right)}{35 \Gamma\left(\frac{7}{10}\right)}\right) \frac{z_{*}^{7} \bar{a}_{\phi}}{z_{+}^{6}}+\frac{5 \sqrt{\pi} \Gamma\left(\frac{8}{5}\right) z_{*}^{11}\left(\bar{a}_{\phi}-1\right)}{66 \Gamma\left(\frac{11}{10}\right) z_{+}^{10}}+\frac{z_{*}^{13} \bar{a}_{\phi}^{2}}{z_{+}^{12}}\left(-\frac{\sqrt{\pi} \Gamma\left(\frac{6}{5}\right)}{25 \Gamma\left(\frac{7}{10}\right)}+\frac{11 \sqrt{\pi} \Gamma\left(\frac{8}{5}\right)}{600 \Gamma\left(\frac{11}{10}\right)}-\frac{4 \sqrt{\pi} \Gamma\left(\frac{9}{5}\right)}{325 \Gamma\left(\frac{13}{10}\right)}\right) \cdots$,

and

$\frac{A}{L_{x}^{4} L^{5}}=\frac{1}{2 \epsilon^{4}}-\frac{\sqrt{\pi} \Gamma\left(\frac{8}{5}\right)}{12 \Gamma\left(\frac{11}{10}\right) z_{*}^{4}}+\frac{z_{*}^{2} \bar{a}_{\phi}}{z_{+}^{6}}\left(\frac{\sqrt{\pi} \Gamma\left(\frac{8}{5}\right)}{30 \Gamma\left(\frac{11}{10}\right)}-\frac{\sqrt{\pi} \Gamma\left(\frac{6}{5}\right)}{5 \Gamma\left(\frac{7}{10}\right)}\right)+\frac{z_{*}^{8} \bar{a}_{\phi}^{2}}{z_{+}^{12}}\left(-\frac{\sqrt{\pi} \Gamma\left(\frac{6}{5}\right)}{25 \Gamma\left(\frac{7}{10}\right)}+\frac{\sqrt{\pi} \Gamma\left(\frac{8}{5}\right)}{100 \Gamma\left(\frac{11}{10}\right)}-\frac{\sqrt{\pi} \Gamma\left(\frac{9}{5}\right)}{50 \Gamma\left(\frac{13}{10}\right)}\right)+\frac{\sqrt{\pi} \Gamma\left(\frac{8}{5}\right) z_{*}^{6}\left(\bar{a}_{\phi}-1\right)}{6 \Gamma\left(\frac{11}{10}\right) z_{+}^{10}} \cdots$

The above expansion can be used to compute corrections in terms of Kaluza-Klein mass and the background gauge field.

HEE is rewritten up to order $l^{6}$ as follows:

$$
\frac{\kappa^{2}}{2 \pi} S=A=\frac{L_{x}^{4} L^{5}}{2 \epsilon^{4}}-\frac{\pi^{5 / 2} \Gamma\left(\frac{8}{5}\right)^{5} L_{x}^{4} L^{5}}{972 \Gamma\left(\frac{11}{10}\right)^{5} l^{4}}+L_{x}^{4} L^{5}\left(-\frac{9 \Gamma\left(\frac{11}{10}\right)^{2} \Gamma\left(\frac{6}{5}\right) \bar{a}_{\phi} l^{2}}{7 \sqrt{\pi} \Gamma\left(\frac{7}{10}\right) \Gamma\left(\frac{8}{5}\right)^{2} z_{+}^{6}}+\frac{729 \Gamma\left(\frac{11}{10}\right)^{5}}{11 \pi^{5 / 2} \Gamma\left(\frac{8}{5}\right)^{5}} \frac{\left(\bar{a}_{\phi}-1\right) l^{6}}{z_{+}^{10}}\right) \cdots
$$

The increased amount of HEE becomes

$$
\Delta S=-\frac{18 \Gamma\left(\frac{11}{10}\right)^{2} \Gamma\left(\frac{6}{5}\right) \bar{a}_{\phi} l^{2} L_{x}^{4} L^{5}}{7 \sqrt{\pi} \Gamma\left(\frac{7}{10}\right) \Gamma\left(\frac{8}{5}\right)^{2} \kappa^{2} z_{+}^{6}} .
$$

The increased amount of energy in the dual field theory is defined as

$$
\Delta E=-\frac{L_{x}^{4} l L^{5} \bar{a}_{\phi}}{2 \kappa^{2} z_{+}^{6}}
$$

Using the relation like the first law $\Delta E=T_{\text {ent }} \Delta S$, the entanglement temperature is defined as

$$
T_{\text {ent }}=\frac{7 \Gamma\left(\frac{7}{10}\right) \Gamma\left(\frac{8}{5}\right)^{2}}{36 \sqrt{\pi} \Gamma\left(\frac{11}{10}\right)^{2} \Gamma\left(\frac{6}{5}\right) l}
$$

\section{APPENDIX B: SECOND ORDER CORRECTION TO HEE}

In this Appendix we would like to extend the study of the first order correction of HEE in the main text to second order. Especially, by virtue of the fact that the FeffermanGraham (FG) expansion is convenient when we consider the asymptotic expansion of the AdS geometry, we compute the second order correction to HEE with a spherical entangling surface in terms of the FG expansion. A general metric in FG gauge is

$$
d s^{2}=\frac{L^{2}}{z^{2}}\left(d z^{2}+g_{\mu \nu}\left(z, x^{\mu}\right) d x^{\mu} d x^{\nu}\right),
$$

where the AdS boundary is located at $z \sim 0$. We approximate that the circle of the $\phi$ direction is large enough
$\left(M_{0} L \ll 1\right)$. Therefore, the boundary metric at $z \rightarrow 0$ is almost flat. Note that the small $M_{0}$ limit is consistent with assumptions of the asymptotic AdS geometry $M_{0} L \ll 1$ and $a_{\phi} L \ll 1$. The metric is

$$
g_{\mu \nu}\left(z, x^{\mu}\right)=\eta_{\mu \nu}+\delta g_{\mu \nu}^{(1)}\left(z, x^{\mu}\right)+\delta g_{\mu \nu}^{(2)}\left(z, x^{\mu}\right) .
$$

We assume that the metric is static. The bulk surface stays at a constant time slice. The embedding scalar is $z=z\left(x^{i}\right)$ only. With the induced metric $h_{i j}=\frac{L^{2}}{z^{2}}\left(g_{i j}+\partial_{i} z \partial_{j} z\right)$, the area is then

$A=\int d^{d-1} x \sqrt{h}=\int d^{d-1} x \frac{L^{d-1}}{z^{d-1}} \sqrt{\operatorname{det} g_{i j}} \sqrt{1+g^{i j} \partial_{i} z \partial_{j} z}$.

To evaluate the leading order correction, we use the solution of the zeroth order $z_{0}=\sqrt{R^{2}-\sum_{i=1}^{d-1}\left(x^{i}\right)^{2}}$.

The first order is

$$
\begin{aligned}
\delta A^{(1)}= & \frac{\alpha}{2} \int d^{d-1} x \sqrt{\operatorname{det} g_{i j}^{(0)}} \\
& \times\left(\sqrt{1+g^{(0) i j} \partial_{i} z_{0} \partial_{j} z_{0}} \operatorname{tr}\left(g^{(0)-1} \delta g^{(1)}\right)\right. \\
& \left.+\frac{\delta g^{(1) i j} \partial_{i} z_{0} \partial_{j} z_{0}}{\sqrt{1+g^{(0) i j} \partial_{i} z_{0} \partial_{j} z_{0}}}\right)
\end{aligned}
$$

where the term linear to $\partial_{i} z_{1}$ vanishes due to the EOM. The second order is 


$$
\begin{aligned}
\delta A^{(2)}= & L^{d-1} \int d^{d-1} x \sqrt{\operatorname{det} g_{i j}^{(0)}}\left\{\frac{2 \alpha^{2} g^{(0) i j} \partial_{i} z_{2} \partial_{j} z_{0}}{2 \sqrt{1+g^{(0) i j} \partial_{i} z_{0} \partial_{j} z_{0}}}+\frac{\alpha^{2} g^{(0) i j} \partial_{i} z_{1} \partial_{j} z_{1}}{2 \sqrt{1+g^{(0) i j} \partial_{i} z_{0} \partial_{j} z_{0}}}\right. \\
& -\left(\frac{\alpha}{2} \delta g^{(1) i j} \partial_{i} z_{0} \partial_{j} z_{0}\right)\left(\frac{2 \alpha g^{(0) i j} \partial_{i} z_{1} \partial_{j} z_{0}}{2\left(1+g^{(0) i j} \partial_{i} z_{0} \partial_{j} z_{0}\right)^{\frac{3}{2}}}\right)+\frac{\alpha^{2}}{2}\left(\frac{2 \delta g^{(1) i j} \partial_{i} z_{1} \partial_{j} z_{0}}{\sqrt{1+g^{(0) i j} \partial_{i} z_{0} \partial_{j} z_{0}}}\right) \\
& +\frac{\alpha^{2}}{2} \frac{\delta g^{(2) i j} \partial_{i} z_{0} \partial_{j} z_{0}}{\sqrt{1+g^{(0) i j} \partial_{i} z_{0} \partial_{j} z_{0}}}-\frac{\alpha^{2}}{8} \frac{\left(\delta g^{(1) i j} \partial_{i} z_{0} \partial_{j} z_{0}\right)^{2}}{\left(1+g^{(0) i j} \partial_{i} z_{0} \partial_{j} z_{0}\right)^{\frac{3}{2}}} \\
& +\frac{\alpha}{2} \operatorname{tr}\left(g^{(0)-1} \delta g^{(1)}\right)\left(\frac{2 \alpha g^{(0) i j} \partial_{i} z_{1} \partial_{j} z_{0}}{2 \sqrt{1+g^{(0) i j} \partial_{i} z_{0} \partial_{j} z_{0}}}+\frac{\alpha}{2} \frac{\delta g^{(1) i j} \partial_{i} z_{0} \partial_{j} z_{0}}{\sqrt{1+g^{(0) i j} \partial_{i} z_{0} \partial_{j} z_{0}}}\right) \\
& \left.+\left(\frac{\alpha^{2}}{2} \operatorname{tr}\left(g^{(0)-1} \delta g^{(2)}\right)-\frac{\alpha^{2}}{4} \operatorname{tr}\left(g^{(0)-1} \delta g^{(1)}\right)^{2}+\frac{\alpha^{2}}{8} \operatorname{tr}^{2}\left(g^{(0)-1} \delta g^{(1)}\right)\right) \sqrt{1+g^{(0) i j} \partial_{i} z_{0} \partial_{j} z_{0}}\right\},
\end{aligned}
$$

where the profile of the minimal surface is corrected due to the change of the bulk metric, and it can be expanded as follows:

$$
z\left(x_{i}\right)=z_{0}\left(x_{i}\right)+\alpha z_{1}\left(x_{i}\right)+\alpha^{2} z_{2}\left(x_{i}\right)+\cdots,
$$

where $z_{0}=\sqrt{R^{2}-\sum_{i=1}^{d-1} x_{i}^{2}}$. Note that since we are only interested in quadratic corrections to the entanglement entropy, $z_{2}$ will not make contributions since it appears linearly in the area functional. By performing the variation of the action, we will obtain the equation of motion for the spherical case.

Following the procedures in [35], from formula (B5) we can divide the second order contribution to three categories by the power of $z_{1}$. In the zeroth order of $z_{1}$,

$$
\begin{aligned}
A_{2,0}= & \int d^{d-1} x L^{d-1} z_{0}^{d}\left[\frac{\alpha^{2}}{2} \operatorname{tr}\left(g^{(0)-1} \delta g^{(2)}\right)-\frac{\alpha^{2}}{4} \operatorname{tr}\left(g^{(0)-1} \delta g^{(1)}\right)^{2}+\frac{\alpha^{2}}{8} \operatorname{tr}^{2}\left(g^{(0)-1} \delta g^{(1)}\right)\right] \frac{R}{z_{0}} \\
= & \int d^{d-1} x L^{d-1} z_{0}^{d} R\left[-\frac{1}{16}\left(1-\frac{r^{2}}{R^{2}(d-1)}\right)\left(T_{00}^{2}+T_{i j} T^{i j}\right)+\frac{T_{i 0} T^{i 0}}{8}\left(1+\frac{r^{2}}{(d-1) R^{2}}\right)\right. \\
& \left.+\frac{x^{i} x^{j} T_{i \alpha} T^{i \alpha}}{4 R^{2}}+\frac{1}{8}\left(T^{2}-T_{x}^{2}-2 T T_{x}\right)\right],
\end{aligned}
$$

where we have made use of $\sqrt{1+g^{(0) i j} \partial_{i} z^{(0)} \partial_{j} z^{(0)}}=R / z^{(0)}$ and

$$
T \equiv T_{i}{ }^{i}, \quad T_{x} \equiv T_{i j} \frac{x^{i} x^{j}}{R^{2}}
$$

The power of $z_{1}$ appears in the second index of $A_{2, n}$. $A_{2,0}$ does not contribute to EOM of $z_{1}$. Next is the power one of $z_{1}$ as follows:

$$
\begin{aligned}
A_{2,1} & =\int d^{d-1} x \frac{L^{d-1}}{z_{0}^{d-1}}\left[-\frac{(d-1) R z_{1} \operatorname{Tr}\left(g_{0}^{-1} g_{1}\right)}{2 z_{0}}+\frac{z_{0}}{R} g^{(1) i j} \partial_{i} z_{1} \partial_{j} z_{1}+\frac{z_{0}}{2 R} \operatorname{Tr}\left(g_{0}^{-1} g_{1}\right) g_{0}^{i j} \partial_{i} z_{0} \partial_{j} z_{1}\right] \\
& =\int d^{d-1} x L^{d-1} \frac{R}{2 z_{0}}\left[T\left(z_{1}-\frac{z_{0}^{2}}{R^{2}} x^{i} \partial_{i} z_{1}\right)+T_{i j}\left(\frac{2 z_{0}^{2} x^{i} \partial^{j} z_{1}}{R^{2}}-\frac{z_{1} x^{i} x^{j}}{R^{2}}-\frac{z_{0}^{2} x^{i} x^{j} x^{k} \partial_{k} z_{1}}{R^{4}}\right)\right]
\end{aligned}
$$

The power two of $z_{1}$ becomes 


$$
\begin{aligned}
A_{2,2} & =\int d^{d-1} x \frac{L^{d-1}}{z_{0}^{d-1}}\left[\frac{z_{0}}{2 R} g^{(0) i j} \partial_{i} z_{1} \partial_{j} z_{1}+\frac{d(d-1)}{2}\left(\frac{z_{1}}{z_{0}}\right)^{2} \frac{R}{z_{0}}+(d-1) \frac{z_{1}}{z_{0}} \frac{x^{i} \partial_{i} z_{1}}{R}\right] \\
& =\int d^{d-1} x \frac{L^{d-1}}{z_{0}^{d}}\left[\frac{d(d-1) z_{1}^{2}}{2 z_{0}^{2}}+\frac{z_{0}^{2}\left(\partial z_{1}\right)^{2}}{2 R^{2}}-\frac{z_{0}^{2}\left(x^{i} \partial_{i} z_{1}\right)^{2}}{2 R^{4}}+\frac{(d-1)}{2} \frac{x^{i} \partial_{i} z_{1}^{2}}{R^{2}}\right]
\end{aligned}
$$

The EOM of $z_{1}$ is obtained from the variation of $A_{2,1}+A_{2,2}$ :

$$
\frac{\delta\left(A_{2,1}+A_{2,2}\right)}{\delta z_{1}}=L^{d-1} \int d^{d-1} x\left\{\frac{R}{2 z_{0}}\left(T-T_{x}\right)+\frac{R}{z_{0}^{d}}\left(\frac{d(d-1) z_{1}}{z_{0}^{2}}+\frac{d-1}{R^{2}} x^{i} \partial_{i} z_{1}\right)\right\}
$$

and

$$
\frac{\delta\left(A_{2,1}+A_{2,2}\right)}{\partial_{k} z_{1}}=L^{d-1} \int d^{d-1} x\left\{\frac{R}{2 z_{0}}\left(-\frac{T z_{0}^{2} x^{k}}{R^{2}}+\frac{2 T_{i}^{k} z_{0}^{2} x^{i}}{R^{2}}-\frac{z_{0}^{2} T_{i j} x^{i} x^{j} x^{k}}{R^{4}}\right)+\frac{R}{z_{0}^{d}\left(\frac{z_{0}^{2} \partial^{k} z_{1}}{R^{2}}-\frac{z_{0}^{2} x^{i} \partial_{i} z_{1} x^{k}}{R^{4}}+(d-1) \frac{z_{1} x^{k}}{R^{2}}\right)}\right\},
$$

so that the equation of motion becomes

$$
\begin{aligned}
& \frac{2-d}{2 R} z_{0} T+\frac{z_{0}(-2-d) T_{x}}{2 R}+\frac{(1-d) z_{1}}{R \cdot z_{0}^{d}}+\frac{\left(z_{0}^{2}-2 R^{2}\right) X^{k} \partial_{k} z_{1}}{R^{3} \cdot z_{0}^{d}}+\frac{\partial_{k} \partial^{k} z_{1}}{z^{d-2} R}-\frac{2\left(x^{k} \partial_{k} z_{1}\left(x^{i} \partial_{i} z_{0}\right)\right)}{R^{3} z_{0}^{d-1}}-\frac{x^{i} x^{k} \partial_{i} \partial_{k} z_{1}}{R^{3} z_{0}^{d-2}} \\
& =\frac{2-d}{2 R} z_{0} T+\frac{z_{0}(-2-d) T_{x}}{2 R}+\frac{(1-d) z_{1}}{z_{0}^{d} R}-\frac{x^{k} \partial_{k} z_{1}}{R^{3} z_{0}^{d-2}}+\frac{\partial_{k} \partial^{k} z_{1}}{R z_{0}^{d-2}}-\frac{x^{i} x^{k} \partial_{i} \partial_{k} z_{1}}{R^{3} \cdot z_{0}^{d-2}}=0,
\end{aligned}
$$

where $z_{0}=\sqrt{R^{2}-\sum_{i}^{d-1} x_{i}^{2}}, T=T_{i}^{i}$ is constant and $T_{x}=T_{i, j} \frac{x^{i} x^{j}}{R^{2}}$. One can set $T_{i}^{i}=T$ for convenience. We just want to solve the $z_{1}$.

We consider an ansatz for $z_{1}$ of the form $\left.z_{1}=T f_{(} r\right)+T_{i, j} x^{i} x^{j} f_{2}(r)$. If we substitute this ansatz, we will have the following equation:

$$
\begin{aligned}
& \frac{T(d-2)}{r} f_{1}^{\prime}+T f_{1}^{\prime \prime}-\frac{T \cdot r^{2}}{R^{2}} f_{1}^{\prime \prime}+2 T f_{2}+\frac{4 T_{x} f_{2}^{\prime} R^{2}}{r}+R^{2} T_{x} f_{2}^{\prime \prime}+\frac{(d-2) R^{2} T_{x} f_{2}^{\prime}}{r}-2 T_{x} f_{2}-4 T_{x} r f_{2}^{\prime}-T_{x} f_{2}^{\prime \prime} r^{2} \\
& \quad=\frac{z_{0}^{d}}{2}\left[(d-2) T+(d+2) T_{x}\right] .
\end{aligned}
$$

Comparing modes $T$ and $T_{x}$, we have the following equation:

$$
\frac{d^{2} f_{2}}{d u^{2}}\left(R^{2}-u^{2}\right)-\frac{d f_{2}}{d u} \frac{(d-1) R^{2}+4 u^{2}}{u}-2 f_{2}=\frac{u^{d}(d+2)}{2},
$$

and

$$
\frac{\left(R^{2}-u^{2}\right)}{R^{2}} \frac{d^{2} f_{1}}{d u^{2}}-\frac{(d-1)}{u} \frac{d f_{1}}{d u}+2 f_{2}=\frac{u^{d}(d-2)}{2} .
$$

We have let $u=z_{0}$, these two equations have the following solution:

$$
f_{1}=-\frac{R^{2} z^{d}}{2(d+1)}, \quad f_{2}=-\frac{z^{d}}{2(d+1)} .
$$

The final answer is to coincide with the second order correction [35] to HEE for spherical entanglement surface. 
[1] C. Holzhey, F. Larsen, and F. Wilczek, Geometric and renormalized entropy in conformal field theory, Nucl. Phys. B424, 443 (1994); P. Calabrese and J. L. Cardy, Entanglement entropy and quantum field theory, J. Stat. Mech. (2004) P002.

[2] P. Calabrese and J. Cardy, Entanglement entropy and conformal field theory, J. Phys. A 42, 504005 (2009); H. Casini and M. Huerta, Entanglement entropy in free quantum field theory, J. Phys. A 42, 504007 (2009).

[3] G. Vidal, J. I. Latorre, E. Rico, and A. Kitaev, Entanglement in Quantum Critical Phenomena, Phys. Rev. Lett. 90, 227902 (2003).

[4] L. Bombelli, R. K. Koul, J. H. Lee, and R. D. Sorkin, A quantum source of entropy for black holes, Phys. Rev. D 34, 373 (1986); M. Srednicki, Entropy and Area, Phys. Rev. Lett. 71, 666 (1993).

[5] S. Ryu and T. Takayanagi, Holographic Derivation of Entanglement Entropy from AdS/CFT, Phys. Rev. Lett. 96, 181602 (2006).

[6] S. Ryu and T. Takayanagi, Aspects of holographic entanglement entropy, J. High Energy Phys. 08 (2006) 045.

[7] T. Nishioka, S. Ryu, and T. Takayanagi, Holographic entanglement entropy: An overview, J. Phys. A 42, 504008 (2009).

[8] T. Nishioka and T. Takayanagi, AdS bubbles, entropy and closed string tachyons, J. High Energy Phys. 01 (2007) 090.

[9] I. R. Klebanov, D. Kutasov, and A. Murugan, Entanglement as a probe of confinement, Nucl. Phys. B796, 274 (2008).

[10] P. V. Buividovich and M. I. Polikarpov, Entanglement entropy in gauge theories and the holographic principle for electric strings, Phys. Lett. B 670, 141 (2008).

[11] D. Dudal and S. Mahapatra, Confining gauge theories and holographic entanglement entropy with a magnetic field, J. High Energy Phys. 04 (2017) 031.

[12] D. Dudal and S. Mahapatra, Interplay between the holographic QCD phase diagram and entanglement entropy, J. High Energy Phys. 07 (2018) 120.

[13] S. Mahapatra, Interplay between the holographic QCD phase diagram and mutual and n-partite information, J. High Energy Phys. 04 (2019) 137.

[14] T. Albash and C. V. Johnson, Holographic studies of entanglement entropy in superconductors, J. High Energy Phys. 05 (2012) 079.

[15] R.-G. Cai, S. He, L. Li, and Y.-L. Zhang, Holographic entanglement entropy in insulator/superconductor transition, J. High Energy Phys. 07 (2012) 088.

[16] R. G. Cai, S. He, L. Li, and Y. L. Zhang, Holographic entanglement entropy on p-wave superconductor phase transition, J. High Energy Phys. 07 (2012) 027.

[17] R. E. Arias and I. S. Landea, Backreacting p-wave superconductors, J. High Energy Phys. 01 (2013) 157.

[18] X. M. Kuang, E. Papantonopoulos, and B. Wang, Entanglement entropy as a probe of the proximity effect in holographic superconductors, J. High Energy Phys. 05 (2014) 130.

[19] M. K. Zangeneh, Y. C. Ong, and B. Wang, Entanglement entropy and complexity for one-dimensional holographic superconductors, Phys. Lett. B 771, 235 (2017).

[20] S. R. Das, M. Fujita, and B. S. Kim, Holographic entanglement entropy of a $1+1$ dimensional p-wave superconductor, J. High Energy Phys. 09 (2017) 016.
[21] Y. Nakagawa, A. Nakamura, S. Motoki, and V. I. Zakharov, Entanglement entropy of SU(3) Yang-Mills theory, Proc. Sci., LAT2009 (2009) 188 [arXiv:0911.2596].

[22] J. Bhattacharya, M. Nozaki, T. Takayanagi, and T. Ugajin, Thermodynamical Property of Entanglement Entropy for Excited States, Phys. Rev. Lett. 110, 091602 (2013).

[23] W. z. Guo, S. He, and J. Tao, Note on entanglement temperature for low thermal excited states in higher derivative gravity, J. High Energy Phys. 08 (2013) 050.

[24] D. Allahbakhshi, M. Alishahiha, and A. Naseh, Entanglement thermodynamics, J. High Energy Phys. 08 (2013) 102.

[25] S. He, D. Li, and J. B. Wu, Entanglement temperature in non-conformal cases, J. High Energy Phys. 10 (2013) 142.

[26] C. Park, Thermodynamic law from the entanglement entropy bound, Phys. Rev. D 93, 086003 (2016).

[27] B. Ning and F. L. Lin, Relative entropy and torsion coupling, Phys. Rev. D 94, 126007 (2016).

[28] A. Ghosh and R. Mishra, Generalized geodesic deviation equations and an entanglement first law for rotating BTZ black holes, Phys. Rev. D 94, 126005 (2016).

[29] Y. Sun and L. Zhao, Holographic entanglement entropies for Schwarzschild and Reisner-Nordström black holes in asymptotically Minkowski spacetimes, Phys. Rev. D 95, 086014 (2017).

[30] A. O'Bannon, J. Probst, R. Rodgers, and C. F. Uhlemann, First law of entanglement rates from holography, Phys. Rev. D 96, 066028 (2017).

[31] A. Bhattacharya and S. Roy, Holographic entanglement entropy and entanglement thermodynamics of "black" nonsusy D3 brane, Phys. Lett. B 781, 232 (2018).

[32] A. Bhattacharya, K. T. Grosvenor, and S. Roy, Entanglement entropy and subregion complexity in thermal perturbations around pure-AdS spacetime, Phys. Rev. D 100, 126004 (2019).

[33] S. F. Lokhande, G. W. J. Oling, and J. F. Pedraza, Linear response of entanglement entropy from holography, J. High Energy Phys. 10 (2017) 104.

[34] E. Caceres, P. H. Nguyen, and J. F. Pedraza, Holographic entanglement chemistry, Phys. Rev. D 95, 106015 (2017).

[35] D. D. Blanco, H. Casini, L. Y. Hung, and R. C. Myers, Relative entropy and holography, J. High Energy Phys. 08 (2013) 060.

[36] J. Lin, M. Marcolli, H. Ooguri, and B. Stoica, Locality of Gravitational Systems from Entanglement of Conformal Field Theories, Phys. Rev. Lett. 114, 221601 (2015).

[37] S. He, J. R. Sun, and H. Q. Zhang, On holographic entanglement entropy with second order excitations, Nucl. Phys. B928, 160 (2018).

[38] X. Dong, Holographic entanglement entropy for general higher derivative gravity, J. High Energy Phys. 01 (2014) 044.

[39] S. S. Pal and S. Panda, Entanglement temperature with Gauss-Bonnet term, Nucl. Phys. B898, 401 (2015).

[40] Y. Sun, H. Xu, and L. Zhao, Thermodynamics and holographic entanglement entropy for spherical black holes in 5D Gauss-Bonnet gravity, J. High Energy Phys. 09 (2016) 060.

[41] P. Bueno, V. S. Min, A. J. Speranza, and M. R. Visser, Entanglement equilibrium for higher order gravity, Phys. Rev. D 95, 046003 (2017). 
[42] F. M. Haehl, E. Hijano, O. Parrikar, and C. Rabideau, Higher Curvature Gravity from Entanglement in Conformal Field Theories, Phys. Rev. Lett. 120, 201602 (2018).

[43] N. I. Gushterov, A. O'Bannon, and R. Rodgers, On holographic entanglement density, J. High Energy Phys. 10 (2017) 137.

[44] E. Witten, Anti-de Sitter space, thermal phase transition, and confinement in gauge theories, Adv. Theor. Math. Phys. 2, 505 (1998).

[45] C. Csaki, H. Ooguri, Y. Oz, and J. Terning, Glueball mass spectrum from supergravity, J. High Energy Phys. 01 (1999) 017.

[46] S. N. Solodukhin, Entanglement entropy, conformal invariance and extrinsic geometry, Phys. Lett. B 665, 305 (2008).

[47] H. Casini, M. Huerta, and R. C. Myers, Towards a derivation of holographic entanglement entropy, J. High Energy Phys. 05 (2011) 036.

[48] H. Liu and M. Mezei, A refinement of entanglement entropy and the number of degrees of freedom, J. High Energy Phys. 04 (2013) 162.

[49] S. A. Hartnoll, Lectures on holographic methods for condensed matter physics, Classical Quantum Gravity 26, 224002 (2009).

[50] V. Balasubramanian and P. Kraus, A stress tensor for anti-de Sitter gravity, Commun. Math. Phys. 208, 413 (1999).

[51] G. T. Horowitz and R. C. Myers, The AdS/CFT correspondence and a new positive energy conjecture for general relativity, Phys. Rev. D 59, 026005 (1998).

[52] M. Henningson and K. Skenderis, The holographic Weyl anomaly, J. High Energy Phys. 07 (1998) 023.

[53] S. de Haro, S. N. Solodukhin, and K. Skenderis, Holographic reconstruction of space-time and renormalization in the AdS/CFT correspondence, Commun. Math. Phys. 217, 595 (2001).
[54] D. Allahbakhshi and M. Alishahiha, Probing fractionalized charges, Adv. High Energy Phys. 2013, 498068 (2013).

[55] M. Fujita, T. Nishioka, and T. Takayanagi, Geometric entropy and Hagedorn/deconfinement transition, J. High Energy Phys. 09 (2008) 016.

[56] I. Bah, L. A. Pando Zayas, and C. A. Terrero-Escalante, Holographic geometric entropy at finite temperature from black holes in global anti-de Sitter spaces, Int. J. Mod. Phys. A 27, 1250048 (2012).

[57] H. Casini and M. Huerta, A finite entanglement entropy and the c-theorem, Phys. Lett. B 600, 142 (2004).

[58] H. Casini and M. Huerta, A c-theorem for the entanglement entropy, J. Phys. A 40, 7031 (2007).

[59] E. H. Lieb and M. B. Ruskai, Proof of the strong subadditivity of quantum-mechanical entropy, J. Math. Phys. (N.Y.) 14, 1938 (1973).

[60] E. H. Lieb and M. B. Ruskai, A Fundamental Property of Quantum-Mechanical Entropy, Phys. Rev. Lett. 30, 434 (1973).

[61] H. Liu and M. Mezei, Probing renormalization group flows using entanglement entropy, J. High Energy Phys. 01 (2014) 098.

[62] S. Kundu and J. F. Pedraza, Aspects of holographic entanglement at finite temperature and chemical potential, J. High Energy Phys. 08 (2016) 177.

[63] M. Ishihara, F. L. Lin, and B. Ning, Refined holographic entanglement entropy for the AdS solitons and AdS black holes, Nucl. Phys. B872, 392 (2013).

[64] B. S. Kim, Entanglement entropy with background gauge fields, J. High Energy Phys. 08 (2017) 041.

[65] N. Birrell and P. Davies, Quantum Fields in Curved Space (Cambridge University Press, Cambridge, England, 2012).

[66] C. P. Herzog and T. Nishioka, Entanglement entropy of a massive fermion on a torus, J. High Energy Phys. 03 (2013) 077. 